\title{
In emulsio selection of a ribozyme that generates free GTP from guanosine and trimetaphosphate
}

\author{
ULRICH F. MULLER, ARVIN AKOOPIE, JOSHUA T. \\ ARRIOLA AND DOUGLAS M. MAGDE
}

University of California, San Diego

Presenting Author: ufmuller@ucsd.edu

Introduction: An early stage of life likely used catalytic RNAs (ribozymes) to support self-replication and a metabolism. Like in today's organisms, nucleoside 5'-triphosphates (NTPs) may have been used as central building blocks for selfreplication and in metabolism. In early stages of life, NTPs could have been generated by reacting nucleoside 5'-hydroxyl groups with the prebiotically plausible molecule trimetaphosphate (Tmp). We previously showed that this chemistry can be catalyzed by ribozymes but these ribozymes generated only RNA 5 '-triphosphates and not free NTPs.

In emulsio selection: To identify ribozymes that generate free NTPs from free nucleosides and Tmp we set up an in vitro selection system in emulsion. Pool molecules with a randomized sequence of 150 nucleotides were incubated with Tmp and the thio-modified nucleotide 6-thio guanosine (6sGsn). Pool molecules catalyzing nucleoside triphosphorylation generated the corresponding 6-thio guanosine triphosphate (6sGTP). The 6sGTP in turn was ligated to the 3 '-terminus of successful pool molecules by a polymerase ribozyme variant. This reaction was carried out in emulsion to prevent the diffusion of 6sGTP to inactive pool molecules. After breaking open the emulsion, thiotagged pool molecules were isolated on mercury containing gels, reverse transcribed, and PCR amplified to initiate a new round of selection. After 13 rounds of selection, the selected pools were analyzed by high throughput sequencing analysis.

Biochemical analysis: Individual sequences from the most highly enriched sequence clusters were analyzed biochemically. The results show that these sequences indeed catalyze the triphosphorylation of guanosine to GTP. The results will be placed into the context of the origin of life. 